\title{
Analysis of Gene Expression in Cyclooxygenase-2-Overexpressed Human Osteosarcoma Cell Lines
}

\author{
Jeong A. Han ${ }^{1}$, Ji-Yeon Kim², Jong-Il Kim³ ${ }^{3,4,5 *}$ \\ ${ }^{1}$ Department of Biochemistry and Molecular Biology, Kangwon National University School of Medicine, Chuncheon 200-701, Korea, \\ ${ }^{2}$ Department of Internal Medicine, Seoul National University Hospital, Seoul 110-744, Korea, \\ ${ }^{3}$ Department of Biochemistry and Molecular Biology, Seoul National University College of Medicine, Seoul 110-799, Korea, \\ ${ }^{4}$ Department of Biomedical Sciences, Seoul National University Graduate School, Seoul 110-799, Korea, \\ ${ }^{5}$ Genomic Medicine Institute, Medical Research Center, Seoul National University, Seoul 110-799, Korea
}

\begin{abstract}
Osteosarcoma is the most common primary bone tumor, generally affecting young people. While the etiology of osteosarcoma has been largely unknown, recent studies have suggested that cyclooxygenase-2 (COX-2) plays a critical role in the proliferation, migration, and invasion of osteosarcoma cells. To understand the mechanism of action of COX- 2 in the pathogenesis of osteosarcoma, we compared gene expression patterns between three stable COX-2-overexpressing cell lines and three control cell lines derived from U2OS human osteosarcoma cells. The data showed that 56 genes were upregulated, whereas 20 genes were downregulated, in COX-2-overexpressed cell lines, with an average fold-change $>1.5$. Among the upregulated genes, COL1A1, COL5A2, FBN1, HOXD10, RUNX2, and TRAPPC2 are involved in bone and skeletal system development, while $D D R 2, R A C 2, R U N X 2$, and TSPAN31 are involved in the positive regulation of cell proliferation. Among the downregulated genes, HIST1H1D, HIST1H2AI, HIST1H3H, and HIST1H4C are involved in nucleosome assembly and DNA packaging. These results may provide useful information to elucidate the molecular mechanism of the COX-2-mediated malignant phenotype in osteosarcoma.
\end{abstract}

Keywords: cell proliferation, cyclooxygenase 2, invasion, osteosarcoma, overexpression, migration

\section{Introduction}

Osteosarcoma is the most common primary bone tumor, representing over $56 \%$ of all bone tumors. It generally affects young people with the age of 15 to 19 and usually occurs in long bones near the metaphyseal growth plates. It has a high tendency to metastasize, and about $10 \%-20 \%$ of patients have macroscopic evidence of metastasis at the time of diagnosis, while $80 \%-90 \%$ of patients are assumed to have micrometastasis. Although it has been postulated that rapid bone growth, exposure to radiation, and genetic predispositions, such as $R B$ or $p 53$ mutations, are thought to be risk factors for developing osteosarcoma, the etiology has not been fully understood [1-3].

Prostaglandin endoperoxide synthase 2 (PTGS2), also called as cyclooxygenase-2 (COX-2), catalyzes the convertsion of arachidonic acid to prostaglandin $\mathrm{H} 2$, from which various prostanoids, including prostaglandin $E_{2}$, are produced [4].

Accumulating evidence indicates that COX-2 is involved in osteosarcoma development and progression. Several studies have reported that high levels of COX-2 expression is associated with advanced clinical stage and metastasis $[5,6]$, as well as with lower overall survival rates and disease-free survival rates [7-9]. In addition, COX-2 inhibition by using RNAi or antisense oligonucleotide inhibits cell proliferation and invasion in human osteosarcoma cells [10, 11]. Also, selective COX-2 inhibitors reduce not only osteosarcoma cell proliferation and invasion in vitro but also tumor growth and metastasis in vivo $[12,13]$. Moreover, we have previously reported that COX-2 overexpression promotes cell 
proliferation, migration, and invasion in U2OS human osteosarcoma cells [14]. These studies strongly suggest that COX-2 might be a causal factor for the development and progression of osteosarcoma. However, the exact mechanisms of action of COX-2 in osteosarcoma are largely unknown.

In an attempt to figure out the mechanism of action of COX-2 in osteosarcoma, we analyzed the gene expression profiles in three COX-2-overexpressed U2OS stable cell lines and three control stable cell lines.

\section{Methods}

\section{Establishment and maintenance of stable cell lines}

Human COX-2 cDNA was subcloned into the pcDNA3 vector containing neo ${ }^{\mathrm{r}}$. U2OS cells were transfected with COX-2 or pcDNA3 DNA using Lipofectamine2000 (Life Technologies, Grand Island, NY, USA). Transfectants were selected in the presence of geneticin, and individual clones were maintained in Dulbecco's modified Eagle's medium, containing fetal bovine serum (10\%), penicillin (100 units $/ \mathrm{mL}$ ), streptomycin (100 units $/ \mathrm{mL}$ ), and geneticin (700 $\mu \mathrm{g} / \mathrm{mL})$, as reported previously [14].

\section{RNA isolation}

Total RNA was extracted from cells with Trizol (Life Technologies), purified with the addition of chloroform, and precipitated with the addition of isopropanol. The RNA concentration was determined by a spectrophotometer, and the quality of RNA was evaluated by the OD $260 / 280$ ratio and gel electrophoresis.

\section{Hybridization to expression arrays}

The following procedures were carried out by Macrogen Co. (Seoul, Korea). First, total RNA was amplified and purified using the Ambion Illumina RNA amplification kit to yield biotinylated cRNA (Ambion, Austin, TX, USA). Briefly, 550 ng of total RNA was reverse-transcribed to cDNA using a T7 oligo(dT) primer. Second-strand cDNA was synthesized, in vitro-transcribed, and labeled with biotin-NTP.
After purification, $750 \mathrm{ng}$ of labeled cRNA was hybridized to the humanHT-12 expression v.4 bead array (Illumina, San Diego, CA, USA) for $16-18 \mathrm{~h}$ at $58^{\circ} \mathrm{C}$. The array signal was detected using Amersham fluorolink streptavidin-Cy3 (GE Healthcare Bio-Sciences, Little Chalfont, UK). Arrays were scanned with an Illumina bead array reader/confocal scanner. Array data were filtered by a detection p-value $<$ 0.05 (similar to signal to noise). Selected gene signal values were log-transformed and normalized by the quantile method.

\section{Statistical analysis}

Basic statistical analyses were performed using Microsoft Excel. Hierarchical cluster analysis was conducted with normalized log2-gene expression values using Cluster 3.0, and the results were visualized using Java Treeview $[15,16]$. An unrooted tree was drawn with $\mathrm{R}$ package. Biological function analysis was performed with official gene names using DAVID (http://david.abcc.ncifcrf.gov/).

\section{Results}

\section{Stable cell lines}

We have previously established stable cell lines overexpressing human COX-2 in U2OS human osteosarcoma cells. To avoid clonal variations, we established three stable COX-2-overexpressing cell lines (U2OS-COX-2 \#1, \#2, and $\# 3$ ) and three control cell lines (U2OS-pcDNA3 \#1, \#2, and \#3). High levels of COX-2 expression were observed by western blot analysis in U2OS-COX-2 cells, whereas the expression was barely detectable in U2OS-pcDNA3 cells. When we cultured the stable cell lines, we observed that cell proliferation, migration, and invasion rates were increased significantly in U2OS-COX-2 cells as compared with U2OS-pcDNA3 cells [14] (Table 1).

\section{Gene expression}

We first performed unsupervised cluster analysis comparing COX-2-overexpressing cell lines and control cell lines. Among 24,371 probe sets, we selected and analyzed 567

Table 1. Summary of stable cell line characteristics

\begin{tabular}{ccccc}
\hline Cell line & Expression level of COX-2 & Proliferation rate & Migration rate (trans-well) & Invasion rate (Matrigel) \\
\hline U2OS-COX-2 \#1 & +++ & +++ & +++ & +++ \\
U2OS-COX-2 \#2 & +++ & +++ & +++ & +++ \\
U2OS-COX-2 \#3 & +++ & +++ & +++ & +++ \\
U2OS-pcDNA3 \#1 & \pm & + & + & + \\
U2OS-pcDNA3 \#2 & \pm & + & + & + \\
U2OS-pcDNA3 \#3 & \pm & + & + & + \\
\hline
\end{tabular}

COX-2, cyclooxygenase-2. 


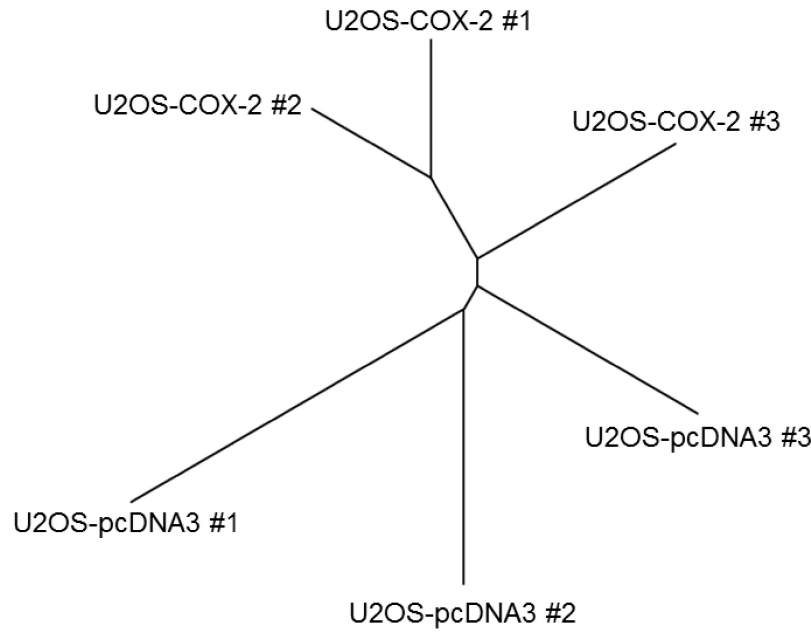

Fig. 1. Segregation between the group of U2OS-COX-2 and the group of U2OS-pcDNA3. Unsupervised cluster analysis was done between six stable cell lines using 567 probe sets with a standard deviation $>0.5$. Normalized $\log _{2}$-gene expression values were used for hierarchical clustering, and an unrooted tree was drawn by $\mathrm{R}$ package. COX-2, cyclooxygenase- 2 . probe sets with a standard deviation $>0.5$. The group of U2OS-COX-2 (U2OS-COX-2 \#1, \#2, and \#3) and the group of U2OS-pcDNA3 (U2OS-pcDNA3 \#1, \#2, and \#3) were well segregated in the unsupervised cluster analysis, confirming that our experiment was validated (Fig. 1).

To identify significantly differentially expressed genes (DEGs) in COX-2-overexpressing osteosarcoma cells, we first performed the Wilcoxon rank sum test between the group of U2OS-COX-2 and the group of U2OS-pcDNA3. The 2,144 probe sets passed the criteria. Then, to identify DEGs, we selected 76 probe sets with an average fold-change $>1.5$.

Among the DEGs, 56 genes were upregulated while 20 genes were downregulated in the group of U2OS-COX-2 as compared to the group of U2OS-pcDNA3 (Tables 2 and 3). The heat map of DEGs is provided in Fig. 2. The gene encoding COX-2, PTGS2, was upregulated by 10.41-fold in the COX-2-overexpressing cell lines as compared to the control cell lines, confirming again that our experiment is

Table 2. Upregulated DEGs in COX-2-overexpressed cell lines

\begin{tabular}{|c|c|c|c|}
\hline Symbol & Description & $\begin{array}{c}\text { Fold } \\
\text { change }\end{array}$ & Accession No. \\
\hline PTGS2 & Prostaglandin-endoperoxide synthase 2 & 10.41 & NM_000963 \\
\hline PAGE2B & $\mathrm{P}$ antigen family, member $2 \mathrm{~B}$ & 4.61 & NM_001015038 \\
\hline COL1A1 & Collagen, type I, alpha 1 & 3.21 & NM_000088 \\
\hline FAP & Fibroblast activation protein, alpha & 2.76 & NM_004460 \\
\hline COL5A2 & Collagen, type $\mathrm{V}$, alpha 2 & 2.35 & NM_000393 \\
\hline LINC00312 & Long intergenic non-protein coding RNA 312 & 2.34 & NR_024065 \\
\hline RAC2 & Ras-related C3 botulinum toxin substrate 2 (rho family, small GTP-binding protein Rac2) & 2.33 & NM_ō_002872 \\
\hline HOXD10 & Homeobox D10 & 2.32 & NM_002148 \\
\hline RCN3 & Reticulocalbin 3, EF-hand calcium-binding domain & 2.07 & NM_020650 \\
\hline FBN1 & Fibrillin 1 & 2.02 & NM_000138 \\
\hline CREB3L1 & CAMP responsive element-binding protein 3 -like 1 & 2.02 & NM_052854 \\
\hline C1orf85 & Chromosome 1 open reading frame 85 & 2.01 & NM_-144580 \\
\hline TSPAN31 & Tetraspanin 31 & 2.01 & NM_005981 \\
\hline DPYSL5 & Dihydropyrimidinase-like 5 & 1.96 & NM_020134 \\
\hline PSMB9 & Proteasome (prosome, macropain) subunit, beta type, 9 , transcript variant 1 & 1.89 & NM_-002800 \\
\hline PSMB9 & Proteasome (prosome, macropain) subunit, beta type, 9, transcript variant 2 & 1.89 & NM_-148954 \\
\hline GPAM & Glycerol-3-phosphate acyltransferase, mitochondrial & 1.89 & NM_020918 \\
\hline NLRP1 & NLR family, pyrin domain-containing 1 , transcript variant 1 & 1.83 & NM_033004 \\
\hline LTBP1 & Latent transforming growth factor beta-binding protein 1 , transcript variant 1 & 1.82 & NM_206943 \\
\hline UNC13A & Unc-13 homolog A (Caenorhabditis elegans) & 1.79 & NM_001080421 \\
\hline VAT1L & Vesicle amine transport 1 -like & 1.78 & NM_020927 \\
\hline MEIS1 & Meis homeobox 1 & 1.77 & NM־ 002398 \\
\hline$D D R 2$ & Discoidin domain receptor family, member 2 , transcript variant 1 & 1.74 & NM_001014796 \\
\hline DYSF & Dysferlin, limb girdle muscular dystrophy 2B (autosomal recessive) & 1.74 & NM_003494 \\
\hline$A C B D 7$ & Acyl-coenzyme A-binding domain-containing 7 & 1.69 & NM_001039844 \\
\hline FMN1 & Formin 1 & 1.68 & NM_001103184 \\
\hline$S C N 3 A$ & Sodium channel, voltage-gated, type III, alpha subunit, transcript variant 1 & 1.68 & NM_006922 \\
\hline NLRP11 & NLR family, pyrin domain-containing 11 & 1.68 & NM_145007 \\
\hline MBOAT2 & Membrane bound O-acyltransferase domain-containing 2 & 1.68 & NM 138799 \\
\hline
\end{tabular}

DEG, differentially expressed gene; COX-2, cyclooxygenase-2. 
Table 2. Continued

\begin{tabular}{|c|c|c|c|}
\hline Symbol & Description & $\begin{array}{c}\text { Fold } \\
\text { change }\end{array}$ & Accession No. \\
\hline MIR196A1 & MicroRNA 196a-1 & 1.67 & NR_029582 \\
\hline ASTN1 & Astrotactin 1, transcript variant 1 & 1.65 & NM_004319 \\
\hline TSHZ2 & Teashirt family zinc finger 2 & 1.64 & NM_173485 \\
\hline MFAP2 & Microfibrillar-associated protein 2, transcript variant 2 & 1.64 & NM_002403 \\
\hline SH3PXD2A & SH3 and PX domains $2 \mathrm{~A}$ & 1.62 & NM_014631 \\
\hline SNORD116-22 & Small nucleolar RNA, C/D box 116-22 & 1.60 & NR_003336 \\
\hline TSHZ2 & Teashirt zinc finger homeobox 2 & 1.59 & NM_001193421 \\
\hline EHD3 & EH domain-containing 3 & 1.59 & NM_014600 \\
\hline$L B H$ & Limb bud and heart development homolog (mouse) & 1.59 & NM_030915 \\
\hline PTPN20B & Protein tyrosine phosphatase, non-receptor type 20B & 1.59 & NM_001042357 \\
\hline C10orf11 & Chromosome 10 open reading frame 11 & 1.59 & NM_032024 \\
\hline SNORD116-2 & Small nucleolar RNA, C/D box $116-2$ on chromosome 15 & 1.59 & NR_003317 \\
\hline EFEMP1 & EGF-containing fibulin-like extracellular matrix protein 1 , transcript variant 1 & 1.59 & NM_en_04105 \\
\hline NAG & Neuroblastoma-amplified protein & 1.58 & NM_015909 \\
\hline HIST4H4 & Histone cluster $4, \mathrm{H} 4$ & 1.58 & NM_175054 \\
\hline KIF5A & Kinesin family member $5 \mathrm{~A}$ & 1.57 & NM_004984 \\
\hline TMEPAI & Transmembrane, prostate androgen-induced RNA, transcript variant 1 & 1.56 & NM_020182 \\
\hline$\cup A C A$ & Vveal autoantigen with coiled-coil domains and ankyrin repeats, transcript variant 1 & 1.55 & NM_018003 \\
\hline RASSF2 & Ras association (RalGDS/AF-6) domain family 2, transcript variant 1 & 1.54 & NM_014737 \\
\hline GPATCH1 & G patch domain containing 1 & 1.54 & NM_018025 \\
\hline LPAR 1 & Lysophosphatidic acid receptor 1 & 1.54 & NM_057159 \\
\hline ARSI & Arylsulfatase family, member I & 1.53 & NM_001012301 \\
\hline TRAPPC2 & Trafficking protein particle complex 2, transcript variant 1 & 1.53 & NM_001011658 \\
\hline CDR1 & Cerebellar degeneration-related protein 1, 34 kDa & 1.53 & NM_004065 \\
\hline WDPCP & WD repeat-containing and planar cell polarity effector & 1.53 & NM_015910 \\
\hline CCDC 186 & Coiled-coil domain-containing protein 186 & 1.52 & NM_018017 \\
\hline RUNX2 & Runt-related transcription factor 2 , transcript variant 1 & 1.51 & NM_001024630 \\
\hline
\end{tabular}

Table 3. Downregulated DEGs in COX-2-overexpressed cell lines

\begin{tabular}{|c|c|c|c|}
\hline Symbol & Description & Fold change & Accession No. \\
\hline HIST1H4C & Histone cluster $1, \mathrm{H} 4 \mathrm{C}$ & 4.63 & NM_003542 \\
\hline HIST $1 \mathrm{H} 3 \mathrm{H}$ & Histone cluster $1, \mathrm{H} 3 \mathrm{~h}$ & 2.05 & NM_003536 \\
\hline HIST1H2AI & Histone cluster 1, H2ai & 1.94 & NM_003509 \\
\hline$C A B Y R$ & Calcium binding tyrosine-(Y)-phosphorylation regulated, transcript variant 1 & 1.91 & NM_012189 \\
\hline FLJ16171 & FLJ16171 protein & 1.90 & NR_046113 \\
\hline$A K R 1 C 2$ & Aldo-keto reductase family 1 , member $\mathrm{C} 2$, transcript variant 2 & 1.89 & NM_205845 \\
\hline$A \cup R K C$ & Aurora kinase $\mathrm{C}$, transcript variant 1 & 1.77 & NM_001015878 \\
\hline HIST1H1D & Histone cluster $1, \mathrm{H} 1 \mathrm{~d}$ & 1.72 & NM_005320 \\
\hline CRLF2 & Cytokine receptor-like factor 2 , transcript variant 1 & 1.70 & NM_001012288 \\
\hline CTAG2 & Cancer/testis antigen 2 , transcript variant 2 & 1.69 & NM_020994 \\
\hline SERPINB5 & Serpin peptidase inhibitor, clade B (ovalbumin), member 5 & 1.65 & NM_002639 \\
\hline$R A R B$ & Retinoic acid receptor, beta, transcript variant 1 & 1.65 & NM_000965 \\
\hline FAT4 & FAT tumor suppressor homolog 4 (Drosophila) & 1.64 & NM_024582 \\
\hline TNFRSF11B & Tumor necrosis factor receptor superfamily, member $11 \mathrm{~b}$ & 1.57 & NM_002546 \\
\hline NOTUM & Notum pectinacetylesterase homolog (Drosophila) & 1.57 & NM_178493 \\
\hline FGF2 & Fibroblast growth factor 2 (basic) & 1.56 & NM_002006 \\
\hline STX11 & Syntaxin 11 & 1.53 & NM_003764 \\
\hline VEPH1 & Ventricular zone-expressed $\mathrm{PH}$ domain homolog 1 (zebrafish) & 1.53 & NM_024621 \\
\hline SPATA31C 1 & SPATA31 subfamily $C$, member 1 & 1.53 & NM_001145124 \\
\hline$A D A M 21$ & ADAM metallopeptidase domain 21 & 1.52 & NM_003813 \\
\hline
\end{tabular}

DEG, differentially expressed gene; COX-2, cyclooxygenase-2. 
validated (Table 2 ).

\section{Functional analysis}

The DEGs were concentrated in several important biological functions

The functions that were most significantly upregulated by

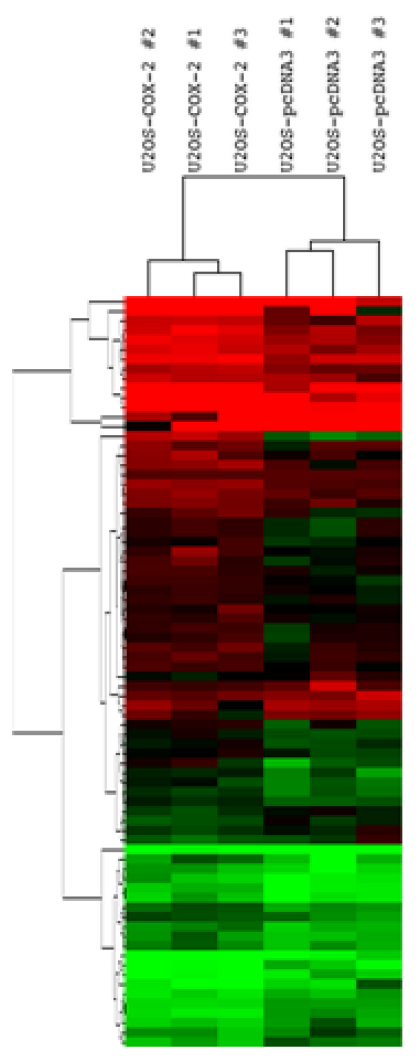

Fig. 2. Gene expression patterns of 76 differentially expressed genes (DEGs). Hierarchical cluster analysis was done between six stable cell lines using 76 DEGs. Normalized $\log _{2}$-gene expression values were analyzed by Cluster 3.0, and the results were visualized using Java Treeview, with red being high and green indicating low.
COX-2 overexpression in U2OS cells were skeletal system development and morphogenesis, bone development and morphogenesis, ossification, and positive regulation of cell proliferation ( $\mathrm{p}<0.05$ ) (Table 4$)$. The genes of COL1A1, COL5A2, FBN1, HOXD10, RUNX2, and TRAPPC2 are involved in skeletal system development and morphogenesis. The genes of COL1A1, COL5A2, and RUNX2 are involved in bone development, morphogenesis and ossification. The genes of DDR2, RAC2, RUNX2, and TSPAN31 are involved in the positive regulation of cell proliferation (Table 4).

The functions that were most significantly downregulated by COX-2 overexpression in U2OS cells were nucleosome assembly, chromatin assembly, and DNA packaging ( $\mathrm{p}<$ 0.05). The genes of HIST1H1D, HIST1H2AI, HIST1H3H, and HIST1H4C are involved in these processes (Table 5).

\section{Discussion}

COX-2 has been reported to promote cell proliferation, migration, and invasion in osteosarcoma cells. COX-2 gene knockdown by RNAi significantly reduced the cell proliferation, migration, and invasion in SaOS2 cells [11], whereas stable expression of COX-2 increased the ability in U2OS cells [14] (Table 1). These studies demonstrated that abnormal COX-2 overexpression is an important determinant for the malignant phenotype of osteosarcoma cells. By using bioinformatics tools, we here provided useful information to understand the underlying mechanism by which COX-2 promotes the malignant phenotype in osteosarcoma cells.

According to our data, the genes encoding extracellular matrix proteins are highly upregulated by COX-2 (Tables 2 and 4). The genes COL1A1, COL5A2, and FBN1 encode $\alpha 1$ chain of type I collagen, $\alpha 2$ chain of type $\mathrm{V}$ collagen, and fibrillin 1, respectively. It has been known that the type I and type $\mathrm{V}$ collagens are major extracellular matrix proteins in

Table 4. Biological functions of upregulated DEGs

\begin{tabular}{lll}
\hline \multicolumn{1}{c}{ Functional category } & \multicolumn{1}{c}{ Genes } & p-value \\
\hline GO_Biological process & & \\
Skeletal system development & PTGS2, FBN1, COL1A1, COL5A2, RUNX2, HOXD10, TRAPPC2 & 0.00009 \\
Ossification & PTGS2, COL1A1, COL5A2, RUNX2 & 0.00248 \\
Bone development & PTCS2, COL1A1, COL5A2, RUNX2 & 0.00300 \\
Positive regulation of cell proliferation & PTGS2, RAC2, TSPAN31, DDR2, RUNX2 & 0.01579 \\
Endochondral ossification & COL1A1, RUNX2 & 0.02803 \\
Skeletal system morphogenesis & COL1A1, RUNX2, HOXD10 & 0.02867 \\
Endochondral bone morphogenesis & COL1A1, RUNX2 & 0.03720 \\
Bone morphogenesis & COL1A1, RUNX2 & 0.04629 \\
GO_Molecular function & & \\
Extracellular matrix structural constituent & FBN1, COL1A1, COL5A2 & 0.01891 \\
\hline
\end{tabular}

DEG, differentially expressed gene. 
Table 5. Biological functions of downregulated DEGs

\begin{tabular}{|c|c|c|}
\hline Functional category & Genes & p-value \\
\hline \multicolumn{3}{|l|}{ GO_Biological process } \\
\hline Nucleosome assembly & HIST1H1D, HIST1H2AI, HIST1H4C, HIST1H3H & 0.00005 \\
\hline Chromatin assembly & HIST1H1D, HIST1H2AI, HIST1H4C, HIST1H3H & 0.00005 \\
\hline Protein-DNA complex assembly & HIST1H1D, HIST1H2AI, HIST1H4C, HIST1H3H & 0.00006 \\
\hline Nucleosome organization & HIST1H1D, HIST1H2AI, HIST1H4C, HIST1H3H & 0.00007 \\
\hline DNA packaging & HIST1H1D, HIST1H2AI, HIST1H4C, HIST1H3H & 0.00013 \\
\hline Chromatin assembly or disassembly & HIST1H1D, HIST1H2AI, HIST1H4C, HIST1H3H & 0.00017 \\
\hline Cellular macromolecular complex assembly & HIST1H1D, HIST1H2AI, HIST1H4C, HIST1H3H & 0.00242 \\
\hline Cellular macromolecular complex subunit organization & HIST1H1D, HIST1H2AI, HIST1H4C, HIST1H3H & 0.00336 \\
\hline Chromatin organization & HIST1H1D, HIST1H2AI, HIST1H4C, HIST1H3H & 0.00395 \\
\hline Chromosome organization & HIST1H1D, HIST1H2AI, HIST1H4C, HIST1H3H & 0.00791 \\
\hline Macromolecular complex assembly & HIST1H1D, HIST1H2AI, HIST1H4C, HIST1H3H & 0.01865 \\
\hline Macromolecular complex subunit organization & HIST1H1D, HIST1H2AI, HIST1H4C, HIST1H3H & 0.02219 \\
\hline \multicolumn{3}{|l|}{ KEGG_Pathway } \\
\hline Systemic lupus erythematosus & HIST1H2AI, HIST1H4C, HIST1H3H & 0.01236 \\
\hline
\end{tabular}

DEG, differentially expressed gene.

bone [17]. Although there is a report that type I collagen promotes cell proliferation and migration in gastric cancer cells [18], the causal relationship between the overexpression of those genes and osteosarcoma has not been investigated well. Therefore, further studies are necessary to elucidate whether overexpression of these genes might be involved in the COX-2-mediated malignant phenotype in osteosarcoma.

Extracellular matrix proteins not only are structural components of the connective tissue in the body but also regulate cell fates through their receptors on the cell surface. DDR2 is one of the receptors for collagen, the expression of which was highly upregulated by COX-2 (Tables 2 and 4). DDR2 is a receptor tyrosine kinase, and its overexpression promotes the migration and invasion of cancer cells derived from breast, head and neck, and prostate [19-21]. Therefore, COX-2 might promote cell proliferation and motility through upregulation of DDR2. Further studies are necessary to elucidate this possibility.

$R A C 2$, a highly upregulated gene by COX-2, is a member of the Rho family of GTPases (Tables 2 and 4). Although there is no report on Rac2 function in osteosarcoma, there is a report that Rac2 GTPase is required for $\mathrm{B}$ cell proliferation and survival [22]. Therefore, COX-2 might promote cell proliferation through upregulation of Rac2 function.

Among the highly upregulated genes, RUNX2 encodes a transcription factor governing osteoblast differentiation and skeletal morphogenesis. Recent studies on Runx2 function in cancer are controversial. Runx2 overexpression reduces cell proliferation in osteosarcoma cell lines [23] but promotes cell motility or invasion in MCF-7 breast cancer cells and PC prostate cancer cells [24, 25]. Therefore, further studies are required to elucidate the function of Runx 2 in osteosarcoma and the relation between COX-2 and Runx2.

Intriguingly, the genes encoding histone proteins are highly downregulated by COX-2 (Tables 3 and 5 ). The genes HIST1H1D, HIST1H2AI, HIST1H3H, and HIST1H4C encode histone $\mathrm{H} 1 \mathrm{H} 1$, histone $\mathrm{H} 2 \mathrm{~A} 1$, histone $\mathrm{H} 3 \mathrm{Al}$, and histone $\mathrm{H} 41$, respectively. As is well known, the nucleosome is the basic unit of DNA packaging, consisting of a core particle and linker DNA. A core particle comprises 147 base pairs of DNA wrapped around a histone octamer (2 copies each of histone $\mathrm{H} 2 \mathrm{~A}, \mathrm{H} 2 \mathrm{~B}, \mathrm{H} 3$, and $\mathrm{H} 4)$. The core particles are connected by 10-90 bp of linker DNA, which could be naked or bound to histone H1 [26]. The nucleosome itself has been known to be a general gene repressor [27, 28]. In addition, RNAimediated knockdown of $\mathrm{H} 2 \mathrm{~A} . \mathrm{Z}$ reduced cell proliferation in bladder cancer cells [29]. These results are compatible with our finding that COX-2 downregulates the expression of histone genes. Therefore, it is worth studying the mechanism of action of histones in osteosarcoma and the mechanism by which COX-2 downregulates histone genes.

Osteosarcoma is a highly malignant tumor, and the 5 -year survival rate is only $20 \%-30 \%$ in patients with macroscopic evidence of metastasis at diagnosis [3]. While the etiology of osteosarcoma has been largely unknown, recent studies have suggested that COX-2 plays a critical role in osteosarcoma development and progression. In this regard, we believe that our study will provide useful information to understand the mechanism of action of COX-2 in osteosarcoma.

\section{Acknowledgments}

This study was supported by 2014 Research Grant from 
Kangwon National University (No. 120141490).

\section{References}

1. He JP, Hao Y, Wang XL, Yang XJ, Shao JF, Guo FJ, et al. Review of the molecular pathogenesis of osteosarcoma. Asian Pac $J$ Cancer Prev 2014;15:5967-5976.

2. Luetke A, Meyers PA, Lewis I, Juergens H. Osteosarcoma treatment - where do we stand? A state of the art review. Cancer Treat Rev 2014;40:523-532.

3. Longhi A, Errani C, De Paolis M, Mercuri M, Bacci G. Primary bone osteosarcoma in the pediatric age: state of the art. Cancer Treat Rev 2006;32:423-436.

4. Smith WL, Garavito RM, DeWitt DL. Prostaglandin endoperoxide $\mathrm{H}$ synthases (cyclooxygenases)-1 and -2. J Biol Chem 1996;271:33157-33160.

5. El-Badawi ZH, Muhammad EM, Noaman HH. Role of immunohistochemical cyclo-oxygenase-2 (COX-2) and osteocalcin in differentiating between osteoblastomas and osteosarcomas. Malays J Pathol 2012;34:15-23.

6. Jiao G, Ren T, Lu Q, Sun Y, Lou Z, Peng X, et al. Prognostic significance of cyclooxygenase-2 in osteosarcoma: a meta-analysis. Tumour Biol 2013;34:2489-2495.

7. Boulytcheva IV, Soloviev YN, Kushlinskii NE, Mahson AN. Expression of molecular markers in the tumor and survival prognosis in osteosarcoma. Bull Exp Biol Med 2010;150:237242.

8. Urakawa H, Nishida Y, Naruse T, Nakashima H, Ishiguro N. Cyclooxygenase- 2 overexpression predicts poor survival in patients with high-grade extremity osteosarcoma: a pilot study. Clin Orthop Relat Res 2009;467:2932-2938.

9. Wang Z, He M, Xiao Z, Wu H, Wu Y. Quantitative assessment of the association of COX-2 (cyclooxygenase-2) immunoexpression with prognosis in human osteosarcoma: a metaanalysis. PLoS One 2013;8:e82907.

10. Wu X, Cai M, Ji F, Lou LM. The impact of COX-2 on invasion of osteosarcoma cell and its mechanism of regulation. Cancer Cell Int 2014;14:27.

11. Zhao Q, Wang C, Zhu J, Wang L, Dong S, Zhang G, et al. RNAi-mediated knockdown of cyclooxygenase2 inhibits the growth, invasion and migration of SaOS2 human osteosarcoma cells: a case control study. J Exp Clin Cancer Res 2011;30:26.

12. Sabino MA, Ghilardi JR, Jongen JL, Keyser CP, Luger NM, Mach DB, et al. Simultaneous reduction in cancer pain, bone destruction, and tumor growth by selective inhibition of cyclooxygenase-2. Cancer Res 2002;62:7343-7349.

13. Naruse T, Nishida Y, Hosono K, Ishiguro N. Meloxicam inhibits osteosarcoma growth, invasiveness and metastasis by COX-2-dependent and independent routes. Carcinogenesis 2006;27:584-592.
14. Lee EJ, Choi EM, Kim SR, Park JH, Kim H, Ha KS, et al. Cyclooxygenase-2 promotes cell proliferation, migration and invasion in U2OS human osteosarcoma cells. Exp Mol Med 2007;39:469-476.

15. de Hoon MJ, Imoto S, Nolan J, Miyano S. Open source clustering software. Bioinformatics 2004;20:1453-1454.

16. Saldanha AJ. Java Treeview: extensible visualization of microarray data. Bioinformatics 2004;20:3246-3248.

17. Shoulders MD, Raines RT. Collagen structure and stability. Annu Rev Biochem 2009;78:929-958.

18. Li A, Zhou T, Guo L, Si J. Collagen type I regulates beta-catenin tyrosine phosphorylation and nuclear translocation to promote migration and proliferation of gastric carcinoma cells. Oncol Rep 2010;23:1247-1255.

19. Zhang K, Corsa CA, Ponik SM, Prior JL, Piwnica-Worms D, Eliceiri KW, et al. The collagen receptor discoidin domain receptor 2 stabilizes SNAIL1 to facilitate breast cancer metastasis. Nat Cell Biol 2013;15:677-687.

20. Yan Z, Jin S, Wei Z, Huilian H, Zhanhai Y, Yue T, et al. Discoidin domain receptor 2 facilitates prostate cancer bone metastasis via regulating parathyroid hormone-related protein. Biochim Biophys Acta 2014;1842:1350-1363.

21. Xu Z, Choudhary S, Voznesensky O, Mehrotra M, Woodard M, Hansen M, et al. Overexpression of COX-2 in human osteosarcoma cells decreases proliferation and increases apoptosis. Cancer Res 2006;66:6657-6664.

22. Walmsley MJ, Ooi SK, Reynolds LF, Smith SH, Ruf S, Mathiot A, et al. Critical roles for Rac1 and Rac2 GTPases in B cell development and signaling. Science 2003;302:459-462.

23. Lucero CM, Vega OA, Osorio MM, Tapia JC, Antonelli M, Stein GS, et al. The cancer-related transcription factor Runx2 modulates cell proliferation in human osteosarcoma cell lines. J Cell Physiol 2013;228:714-723.

24. Baniwal SK, Khalid O, Gabet Y, Shah RR, Purcell DJ, Mav D, et al. Runx2 transcriptome of prostate cancer cells: insights into invasiveness and bone metastasis. Mol Cancer 2010;9:258.

25. Leong DT, Lim J, Goh X, Pratap J, Pereira BP, Kwok HS, et al. Cancer-related ectopic expression of the bone-related transcription factor RUNX2 in non-osseous metastatic tumor cells is linked to cell proliferation and motility. Breast Cancer Res 2010;12:R89.

26. Richmond TJ, Davey CA. The structure of DNA in the nucleosome core. Nature 2003;423:145-150.

27. Han M, Grunstein M. Nucleosome loss activates yeast downstream promoters in vivo. Cell 1988;55:1137-1145.

28. Lorch Y, LaPointe JW, Kornberg RD. Nucleosomes inhibit the initiation of transcription but allow chain elongation with the displacement of histones. Cell 1987;49:203-210.

29. Kim K, Punj V, Choi J, Heo K, Kim JM, Laird PW, et al. Gene dysregulation by histone variant H2A.Z in bladder cancer. Epigenetics Chromatin 2013;6:34. 